\title{
Mutation in human selenocysteine transfer RNA selectively disrupts selenoprotein synthesis
}

\author{
Erik Schoenmakers, ${ }^{1}$ Bradley Carlson, ${ }^{2}$ Maura Agostini, ${ }^{1}$ Carla Moran, ${ }^{1}$ Odelia Rajanayagam, ${ }^{1}$ Elena Bochukova, ${ }^{1}$ Ryuta Tobe, ${ }^{2}$ \\ Rachel Peat, ${ }^{3}$ Evelien Gevers, ${ }^{4}$ Francesco Muntoni, ${ }^{5}$ Pascale Guicheney, ${ }^{3}$ Nadia Schoenmakers, ${ }^{1}$ Sadaf Farooqi, ${ }^{1}$ Greta Lyons, ${ }^{1}$ \\ Dolph Hatfield, ${ }^{2}$ and Krishna Chatterjee ${ }^{1}$
}

'Wellcome Trust-MRC Institute of Metabolic Science, University of Cambridge, Cambridge, United Kingdom. ${ }^{2}$ Molecular Biology of Selenium Section, Mouse Cancer Cenetics Program, Center for Cancer Research, NIH, Bethesda, Maryland, USA. ${ }^{3}$ INSERM, UMR S1166, Institute of Cardiometabolism and Nutrition (ICAN), Sorbonne Universités, Université Pierre et Marie Curie, University of Paris 06, Paris, France. ${ }^{4}$ Department of Paediatric Endocrinology, Royal London Hospital, London, United Kingdom. ${ }^{5}$ Dubowitz Neuromuscular Centre, University College London Institute of Child Health, London, United Kingdom.

\begin{abstract}
Selenium is a trace element that is essential for human health and is incorporated into more than $\mathbf{2 5}$ human selenocysteinecontaining (Sec-containing) proteins via unique Sec-insertion machinery that includes a specific, nuclear genome-encoded, transfer RNA (tRNA ${ }^{[\mathrm{Ser}] \mathrm{Sec}}$ ). Here, we have identified a human tRNA ${ }^{[\mathrm{Ser}] \mathrm{Sec}}$ mutation in a proband who presented with a variety of symptoms, including abdominal pain, fatigue, muscle weakness, and low plasma levels of selenium. This mutation resulted in a marked reduction in expression of stress-related, but not housekeeping, selenoproteins. Evaluation of primary cells from the homozygous proband and a heterozygous parent indicated that the observed deficit in stress-related selenoprotein production is likely mediated by reduced expression and diminished 2'-0-methylribosylation at uridine 34 in mutant tRNA ${ }^{[\mathrm{Ser}] \mathrm{Sec}}$. Moreover, this methylribosylation defect was restored by cellular complementation with normal tRNA $^{[\text {[Ser]Sec }}$. This study identifies a tRNA mutation that selectively impairs synthesis of stress-related selenoproteins and demonstrates the importance of tRNA modification for normal selenoprotein synthesis.
\end{abstract}

\section{Introduction}

The 21st proteinogenic amino acid, selenocysteine (Sec), is a constituent of more than 25 human selenoproteins. Sec incorporation requires UGA codons in selenoprotein mRNAs to be decoded as Sec, preventing their usual interpretation as termination codons targeting many mRNAs for nonsense-mediated decay (NMD) (1, 2). This is achieved via a unique, highly conserved, Sec-insertion machinery comprising trans-acting factors (e.g., SECIS binding protein 2 [SECISBP2], Sec transfer RNA-specific [tRNA-specific] eukaryotic elongation factor [EEFSEC], tRNA $\left.{ }^{[\mathrm{Ser}] \mathrm{Sec}}\right)$ interacting with cis-acting Sec-insertion sequence (SECIS) elements, located in the 3' UTR of most eukaryotic selenoprotein mRNAs (Figure 1). Mutations in SECISBP2 cause a multisystem disorder with myopathic features due to selenoprotein N (SEPN1) deficiency, increased ROS attributable to lack of antioxidant selenoenzymes - glutathione peroxidases (GPxs) and thioredoxin reductases (TrxRs) - and thyroid dysfunction secondary to loss of selenoprotein deiodinases $(3,4)$; O-phosphoseryl-tRNA:Sec tRNA synthase (SEPSECS) defects cause progressive cerebellocerebral atrophy, likely reflecting global disruption of selenoprotein synthesis $(5,6)$.

\section{Results and Discussion}

An 8-year-old male (the proband), investigated for symptoms including abdominal pain, fatigue, and muscle weakness, exhib-

Conflict of interest: The authors have declared that no conflict of interest exists. Submitted: September 18, 2015; Accepted: December 18, 2015.

Reference information: J Clin Invest. 2016;126(3):992-996. doi:10.1172/JCI84747. ited thyroid dysfunction (raised T4, normal T3, raised reverse T3) suggestive of impaired deiodinase activity in combination with low plasma selenium levels, reflecting deficiencies of circulating red cell GPx, plasma GPx, and selenoprotein P (SEPP1) (Table 1 and Figure 2B), as seen in cases with SECISBP2 mutations. Muscle imaging showed mild signal intensity change (Supplemental Figure 1A; supplemental material available online with this article; doi:10.1172/ JCI84747DS1), with negligible SEPN1 expression in his dermal fibroblasts (Figure 2B). Endogenous $\mathrm{H}_{2} \mathrm{O}_{2}$ levels in fibroblasts from the proband and a SECISBP2-deficient patient were comparably elevated, consistent with impaired antioxidant defence in both contexts (Supplemental Figure 1B). However, comparison of selenoprotein expression profiles revealed significant differences, with preservation of housekeeping selenoproteins (e.g., TrxRs, Figure 2, $A$ and B; GPx4, Figure 2B) in cells from the proband compared with SECISBP2 deficiency cases. In contrast, expression of stress-related selenoproteins (e.g., GPx1, GPx3, SEPW1) was similarly reduced in both contexts. Furthermore, comparison of mRNA expression patterns indicated markedly reduced transcript levels for some selenoproteins in SECISBP2 deficiency cases, consistent with known propensity to NMD-mediated mRNA instability in this context (1, 4), whereas selenoprotein mRNA levels in the proband were either normal or slightly increased (Figure 2C). These findings, together with normal SECISBP2 protein expression in the proband (Figure 2B) and failure to identify abnormalities with SECISBP2 sequencing, suggested a defect elsewhere in the Sec-insertion pathway.

Homozygosity mapping in this family with known parental consanguinity identified a single interval in the proband, encom- 


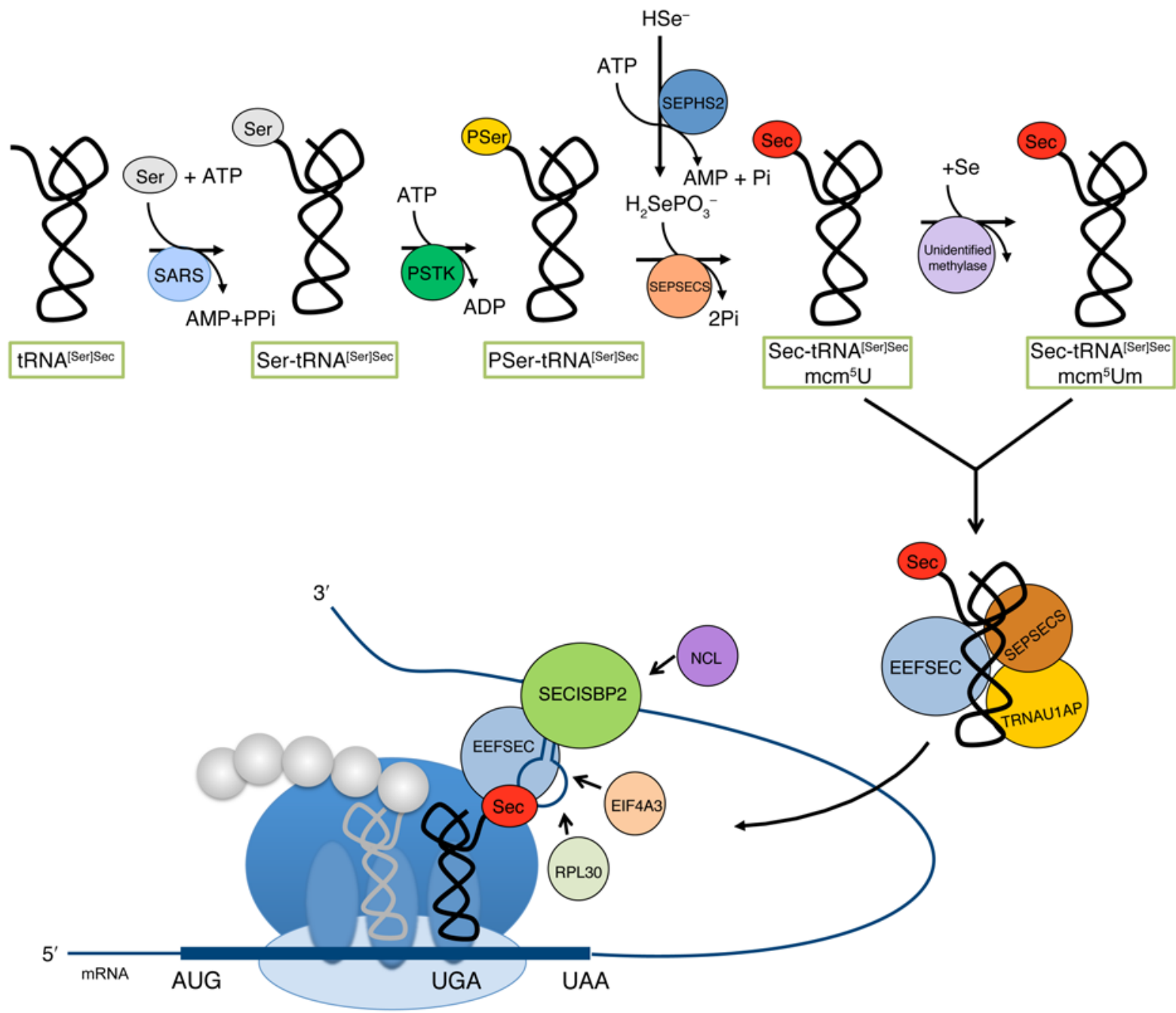

Figure 1. Pathways of Sec synthesis and incorporation into selenoproteins. The synthesis of Sec (upper pathway) occurs on its tRNA, with initial

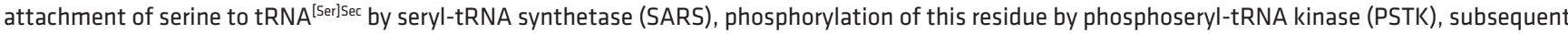
conversion of this phosphoserine (PSer) to an intermediate by SEPSECS, and acceptance of selenophosphate to generate Sec-tRNA ${ }^{[\mathrm{Ser}] \mathrm{Sec}} \mathrm{mcm}^{5} \mathrm{U}$. Selenophosphate is synthesized from selenide and ATP by selenophosphate synthetase 2 (SEPHS2) (2); Sec-tRNA ${ }^{\text {[Ser]Sec }} \mathrm{mcm}^{5} \mathrm{U}$ is partially methylated by an unidentified Um34 methylase, and the level of methylation is dependent on selenium status (7, 9). A multiprotein complex containing tRNA selenocysteine 1 associated protein 1 (TRNAU1AP), EEFSEC, and Sec-tRNA ${ }^{[\mathrm{Ser}] \text { Sec }}$ isoform by SECISBP2, wherein SECISBP2 is bound to the SECIS stem loop element located in the $3^{\prime}$ UTR of mammalian selenoprotein mRNAs decoding UCA Sec codons at the ribosomal acceptor site to mediate Sec incorporation into nascent polypeptide. Other factors, such as ribosomal protein L30 (RPL30), eukaryotic translation initiation factor 4A3 (EIF4A3), and nucleolin (NCL), also regulate the Sec insertion process $(1,2,7)$.

passing the chromosomal locus of only 1 gene (TRU-TCA1-1) in the Sec-incorporation pathway, encoding tRNA ${ }^{[\mathrm{Ser}] \mathrm{Sec}}$ (Figure $3 \mathrm{~A})$. Sequencing of this single-copy gene (Supplemental Table 1) in the proband indicated homozygosity for a single nucleotide change (C65G) (Figure 3B); the nucleotide change (not found in 60,000 exome [Exome Aggregation Consortium (ExAc)] and other published data sets) segregated with phenotype, with unaffected parents and 1 sibling being heterozygous (Table 1). Additional nucleotide changes outside the coding region corresponded to recognized SNPs that did not segregate with phenotype (Supplemental Figure 2).

Quantitative analyses in primary cells from the homozygous proband and heterozygous parent indicated generation of both WT and C65G mutant tRNA ${ }^{[\mathrm{Ser}] \mathrm{Sec}}$, but reduced mutant transcript levels (Supplemental Figure 3). Following transcription, $t R N A^{[S e r] S e c}$ undergoes maturation with sequential base modifications, yielding 2 major tRNA ${ }^{[\mathrm{Ser}] \mathrm{Sec}}$ isoforms containing either 5-methoxycarbonylmethyluridine $\left(\mathrm{mcm}^{5} \mathrm{U}\right)$ or 5-methoxycarbonylmethyl-2'-Omethyluridine $\left(\mathrm{mcm}^{5} \mathrm{Um}\right)$ at position 34 , situated in the anticodon loop (Figure 3B), possibly affecting codon-anticodon interaction in the ribosomal complex. The relative preponderance of these isoforms is influenced by systemic selenium status (7-9), and each subtype has a differing role in selenoprotein synthesis. Synthesis of housekeeping selenoproteins (e.g., TrxR1, TrxR3, GPx4) is dependent on the $\mathrm{mcm}^{5} \mathrm{U}$ isoform, whereas production of stressrelated selenoproteins (e.g., GPx1, GPx3, SEPW1) is directed by the $\mathrm{mcm}^{5} \mathrm{Um}$ species $(1,8)$. These $2 \mathrm{tRNA}^{[\text {[ser]Sec }}$ populations were quantitated and resolved chromatographically in patient-derived 

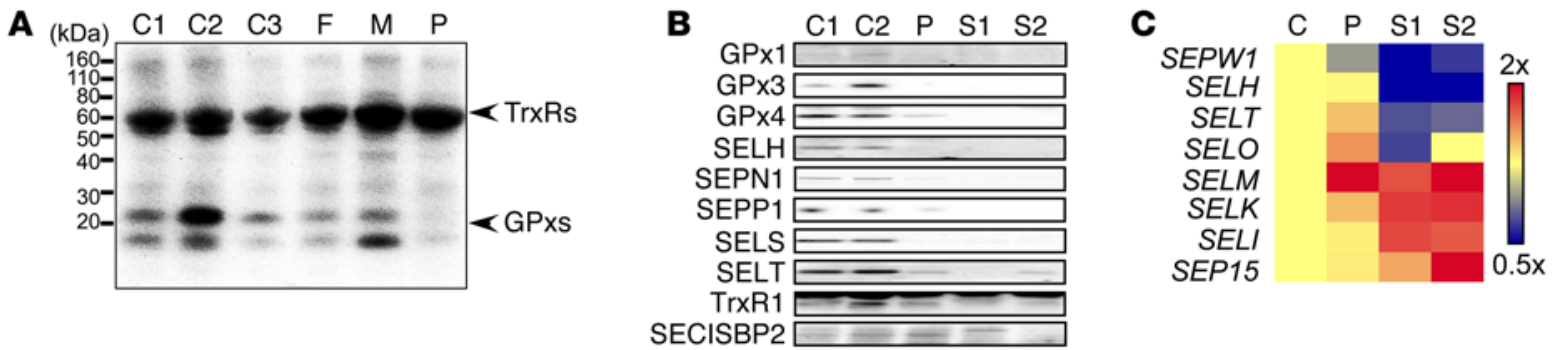

Figure 2. Disorder with distinctively abnormal selenoprotein profile. (A) Autoradiograph of ${ }^{75}$ Se-radiolabeled peripheral blood mononuclear cells from proband $(P)$, father $(F)$, mother $(M)$, and controls $(C 1, C 2, C 3)$, with position of relevant selenoproteins marked by arrowheads. A representative experiment, performed at least twice, from samples taken on 1 occasion. (B) Western blots of selenoproteins and SECISBP2 in dermal fibroblasts or plasma (GPx3, SEPP1 lanes) from controls, proband, and patients with known SECISBP2 mutations (S1, S2). (C) Heat map comparing selenoprotein mRNA expression levels in fibroblasts from control, uniformly designated yellow, with proband and SECISBP2-deficient patients. A representative experiment, performed at least twice, from 1 sample set.

primary cells. Compared with the control, $\mathrm{mcm}^{5} \mathrm{U}$ levels (peak I) were virtually unchanged, with some reduction of $\mathrm{mcm}^{5} \mathrm{Um}$ (peak II) in the heterozygous parent; in the proband, disproportionately greater (5-fold) diminution in tRNA ${ }^{[S e r] S e c} \mathrm{mcm}^{5} \mathrm{Um}$ levels was observed (Figure 3C).

The final step in tRNA ${ }^{[\mathrm{Ser}] \mathrm{Sec}}$ maturation, with methylation of $\mathrm{mcm}^{5} \mathrm{U}$ at position $\mathrm{U} 34$, requires other prior base modifications (e.g., pseudouridine $[\mathrm{p} \psi]$ and 1-methyladenosine $[\mathrm{pm} 1 \mathrm{~A}]$ at positions 55 and 58, respectively) (7), intact secondary and tertiary structures (10), and aminoacylation of tRNA ${ }^{[\mathrm{Ser}] \mathrm{Sec}}$ (11). To investigate base modifications, radiolabeled tRNA ${ }^{[\mathrm{Ser}][\mathrm{sec}}$ transcripts were injected into Xenopus oocytes and analyzed chromatographically, indicating significantly attenuated modifications $\left(\mathrm{mcm}^{5} \mathrm{Um}\right.$ at position U34 [peak III in Supplemental Figure 4B] and i6A at position 37 [peak III in Supplemental Figure 4A]) in C65G tRNA ${ }^{[\mathrm{Ser}] \mathrm{Sec}}$ compared with WT, confirming that maturation of mutant tRNA ${ }^{[\mathrm{Ser}] \mathrm{Sec}}$ is indeed impaired. Additional studies showed that the $\mathrm{C} 65 \mathrm{G}$ tRNA ${ }^{[\mathrm{Ser}] \mathrm{Sec}}$ mutation does not perturb initial tRNA ${ }^{[\mathrm{Ser}] \mathrm{Sec}}$ aminoacylation with serine or Sec synthesis and may even enhance binding to SEPSECS (Supplemental Figure 5, A-C, and see Figure 1); thus, major structural differences between mutant and WT tRNA ${ }^{[\mathrm{Ser}] \mathrm{Sec}}$, which would have abrogated these early steps in tRNA ${ }^{[\mathrm{Ser}] \mathrm{sec}}$ generation, are unlikely.

In complementation studies, expression of WT tRNA ${ }^{[s][S e c}$ (Figure 3D, middle panel, lane 2), but not SECISBP2 (Figure 3D, middle panel, lane 3), in dermal fibroblasts from the proband restored selenoprotein synthesis. Conversely, introduction of SECISBP2 corrected diminished selenoprotein levels in cells from a SECISBP2-deficient patient (4) (Figure 3D, right panel, lane 3), whereas WT tRNA ${ }^{[\mathrm{Ser}] S \mathrm{se}}$ expression was ineffective in this context (Figure 3D, right panel, lane 2).

We have described what we believe is the first mutation in a nuclear genome-encoded, human tRNA associated with a multisystem clinical phenotype and selectively impaired biosynthesis of stress-related, but not housekeeping, selenoproteins. Our results indicate that early steps (aminoacylation, Sec generation) in tRNA ${ }^{[\text {Ser]Sec }}$ synthesis are unperturbed (Supplemental Figure 5). In the heterozygous father, the tRNA ${ }^{[\mathrm{Ser}] \mathrm{Sec}}$ population was reduced by approximately $40 \%$ and comprised approximately $74 \%$ WT and $26 \%$ mutant tRNAs; in the proband, mutant tRNA ${ }^{[S e r] S e c}$ levels were reduced approximately 70\% (Figure 3C and Supplemental Figure 3). The basis for such reduction in mutant tRNA ${ }^{[\text {Ser]Sec }}$ levels is unclear, but possibilities include anomalous transcription and greater mutant transcript turnover or transcript degradation secondary to defective mutant tRNA ${ }^{[\mathrm{Ser}] \mathrm{Sec}}$ maturation.

Preserved selenoprotein levels in heterozygous parents (Figure $2 \mathrm{~A}$ ) suggest that moderate reduction in $\mathrm{TRNA}^{\text {[Ser]Sec }}$ levels is not rate limiting for selenoprotein biosynthesis, as has been documented in other contexts (8). However, in the proband, much lower levels of tRNA ${ }^{\text {[Ser]Sec }}$ were sufficient for effective synthesis of some housekeeping (e.g., TrxRs), but not stress-related, selenoproteins. Interestingly, comparing the proband with SECISBP2-defect cases, synthesis of the essential selenoprotein GPx4 was preserved, but severely impaired (Figure 2B). Mice lacking the $\mathrm{mcm}^{5} \mathrm{Um}$ isoform exhibit reduced GPx4 levels, suggesting that both tRNA ${ }^{[\mathrm{Ser}] \mathrm{Sec}}$ isoforms are required for its synthesis (2); we suggest that 5-fold decreased tRNA ${ }^{[\mathrm{Ser}] \mathrm{Sec}} \mathrm{mcm}^{5} \mathrm{Um}$ in the proband (Figure $3 \mathrm{C}$ and Supplemental Figure 4) also contributed to reduced GPx4 in his case. 

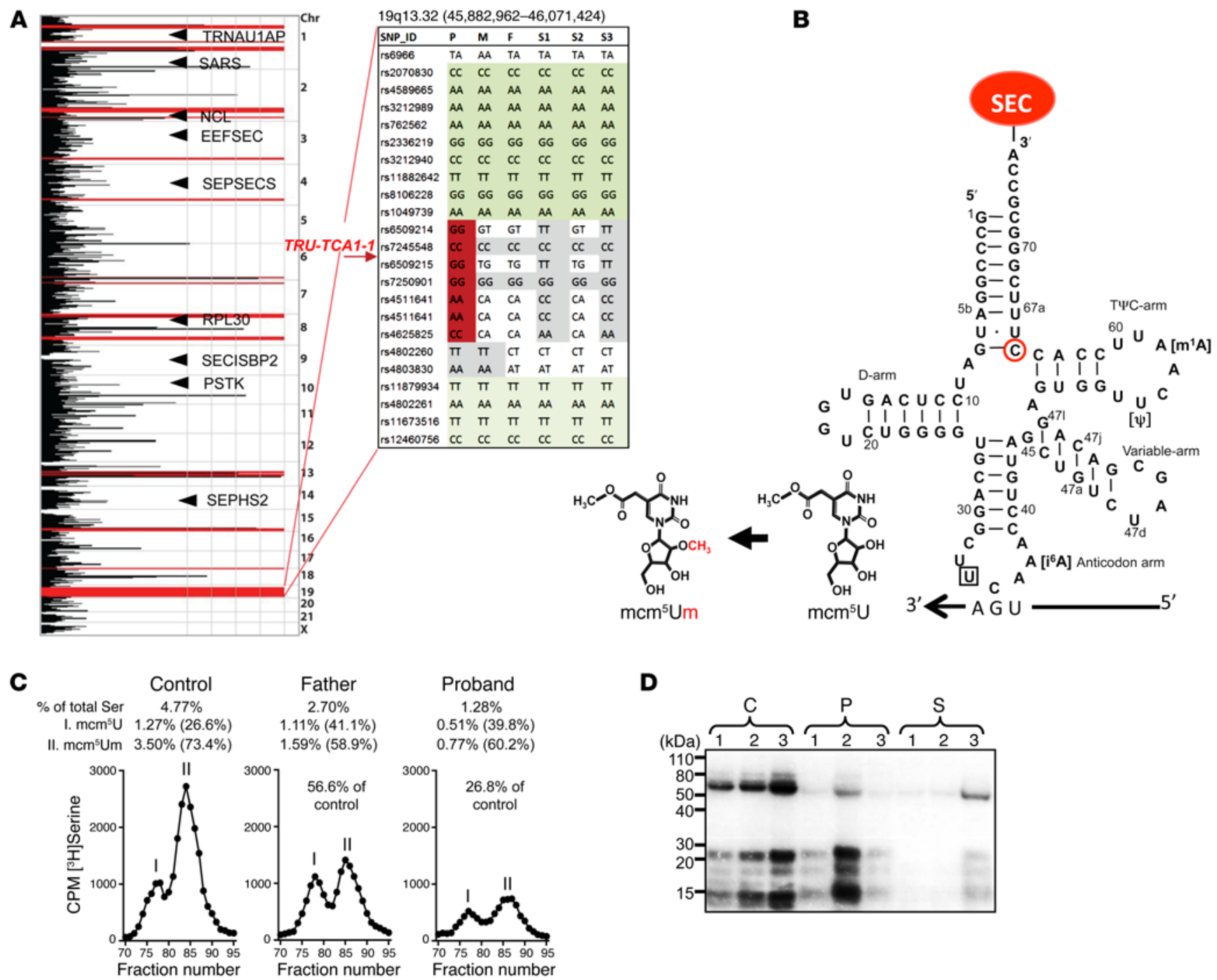

Fraction number

Figure 3. Identification, functional analysis, and complementation of tRNA ${ }^{[\mathrm{Ser}] \mathrm{Sec}}$ mutation. (A) Representation of SNP mapping in proband visualizes runs of homozygosity (ROH) (red peaks) across the genome, with the locations of selenoprotein biosynthetic pathway genes overlaid (TRU-TCA1-1: transfer RNA-Sec [TCA] 1-1). A single ROH overlaps TRU-TCA1-1, and the inset tabulates SNP genotypes in family members (siblings: S1, S2, S3) encompassing this gene locus. (B) Clover leaf model of Sec-tRNA ${ }^{[\mathrm{Ser}] \mathrm{Sec}}$ with the mutated base (ringed) and uridine (boxed) that is modified to Sec-tRNA ${ }^{[\mathrm{Ser}] \mathrm{Sec}} \mathrm{mcm}^{5} \mathrm{U}$ and Sec-tRNA ${ }^{[\mathrm{Ser}] \mathrm{sec}} \mathrm{mcm}^{5} \mathrm{Um}_{\mathrm{m}}$ depicted. (C) Chromatographic elution profiles and quantitation of total tRNA ${ }^{[S e r] S e c}$ as well as $\mathrm{mcm}^{5} \mathrm{U}$ (peak I) and $\mathrm{mcm}^{5} \mathrm{Um}$ (peak II) isoforms in cells from control, father, or proband. (D) Autoradiograph of ${ }^{75} \mathrm{Se}$-labeled primary skin fibroblasts from control, proband, or SECISBP2-deficient subjects transfected with either empty (lane 1), tRNA ${ }^{[\text {Ser]Sec }}$ (lane 2), or SECISBP2 (lane 3) expression vectors. One representative experiment, performed at least three times, is shown.

In patients with global selenoprotein deficiency due to defective SECISBP2, many features (e.g., photosensitivity, agedependent hearing loss) are attributed to ROS-mediated damage, secondary to loss of antioxidant defence (4). It remains to be seen whether such phenotypes are ameliorated in our mutant tRNA ${ }^{[\mathrm{Ser}] \mathrm{Sec}}$ patient, in whom synthesis of some antioxidant selenoenzymes (e.g., TrxRs, GPx4) is preserved. It is well recognized that systemic selenium status influences relative proportions of mutant tRNA ${ }^{[\mathrm{Ser}] \mathrm{Sec}}$ isoforms, where $\mathrm{mcm}^{5} \mathrm{Um}$ is enriched in the Se-replete state and poorly expressed in Se deficiency (7-9). Should careful studies altering the selenium status of patient-derived cells in vitro verify that selective selenoprotein deficits can indeed be corrected without undue toxicity, intervention with selenium supplementation in vivo could be a therapeutic possibility.

\section{Methods}

Further methods are detailed in Supplemental Methods.

Statistics. In studies in which statistical analyses were performed, a 2-tailed Student's $t$ test was used to generate $P$ values. $P$ values less than or equal to 0.05 were considered significant.

Study approval. All investigations were part of an ethically approved protocol (Cambridgeshire LREC 98/154) and/or were clinically indicated and undertaken with prior informed patient consent.

\section{Author contributions}

ES, DH, and KC designed the study. CM, EG, FM, NS, and GL recruited and clinically characterized patients. EB and SF undertook SNP genotyping and bioinformatic analysis of data. OR performed Sanger sequence analysis and interpretation of data. ES, MA, RP, and PG quantified selenoproteins. ES, BC, and RT ana- 
lyzed tRNA ${ }^{[S e r] S e c}$ function. ES, DH, and KC prepared the draft manuscript. All authors contributed to discussion of the results and edited and approved the final version.

\section{Acknowledgments}

Our research is supported by funding from the Wellcome Trust (100585/Z/12/Z to N. Schoenmakers, 095564/Z/11/Z to K. Chatterjee), the NIH Research Biomedical Research Centre Cambridge (to C. Moran, N. Schoenmakers, and K. Chatterjee) or
Great Ormond Street (to F. Muntoni) and the Intramural Research Program of the Center for Cancer Research, National Cancer Institute, NIH (to D.L. Hatfield).

Address correspondence to: Krishna Chatterjee, University of Cambridge, Metabolic Research Laboratories, Institute of Metabolic Science, Level 4, Box 289, Addenbrooke's Hospital, Cambridge, CB2 OQQ, United Kingdom. Phone: 44.1223.336842; E-mail:kkc1@medschl.cam.ac.uk.
1. Shetty SP, Copeland PR. Selenocysteine incorporation: A trump card in the game of mRNA decay. Biochemie. 2015;114:97-101.

2. Labunskyy VM, Hatfield DL, Gladyshev VN. Selenoproteins: molecular pathways and physiological roles. Physiol Rev. 2014;9(3):739-777.

3. Dumitrescu AM, et al. Mutations in SECISBP2 result in abnormal thyroid hormone metabolism. Nat Genet. 2005;37(11):1247-1252.

4. Schoenmakers E, et al. Mutations in the selenocysteine insertion sequence-binding protein 2 gene lead to a multisystem selenoprotein deficiency disorder in humans. JClin Invest. 2010;120(12):4220-4235.
5. Agamy O, et al. Mutations disrupting selenocysteine formation cause progressive cerebello-cerebral atrophy. Am J Hum Genet. 2010;87(4):538-544.

6. Anttonen AK, et al. Selenoprotein biosynthesis defect causes progressive encephalopathy with elevated lactate. Neurology. 2015;85(4):306-315.

7. Diamond AM, et al. Dietary selenium affects methylation of the wobble nucleoside in the anticodon of selenocysteine tRNA([Ser]Sec). J Biol Chem. 1993;268(19):14215-14223.

8. Carlson BA, Yoo M, Tsuji PA, Gladyshev VM, Hatfield DL. Mouse models targeting selenocysteine tRNA expression for elucidating the role of selenoproteins in health and development. Molecules.
2009;14(9):3509-3527.

9. Hatfield D, Lee BJ, Hampton L, Diamond AM. Selenium induces changes in the selenocysteine tRNA[Ser]Sec population in mammalian cells. Nucleic Acids Res. 1991;19(4):939-943.

10. Kim LK, et al. Methylation of the ribosyl moiety at position 34 of selenocysteine tRNA[Ser]Sec is governed by both primary and tertiary structure. RNA. 2000;6(9):1306-1315.

11. Kim JY, et al. Inhibition of selenocysteine tRNA[Ser]Sec aminoacylation provides evidence that aminoacylation is required for regulatory methylation of this tRNA. Biochem Biophys Res Commun. 2011;409(4):814-819. 